\author{
Military Technical College \\ Kobry Elkobbah, Cairo, \\ Egypt.
}

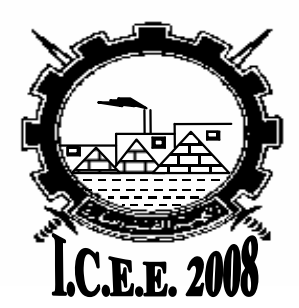

$4^{\text {th }}$ International Conference

On

Chemical \& Environmental

Engineering

27-29 May 2008

\title{
ANALYSIS OF EXPLOSIVE RESIDUES AND POST EXPLOSION ANALYSIS
}

\author{
Amer A.A. ${ }^{*}$, Radwan M. A. ${ }^{*}$, Ismail M.M.* , El-basuney S.S.*,
}

\begin{abstract}
One of the more challenging areas in the field of explosives is the analysis of explosive residues. The detection and identification of explosive traces on individuals, on clothing, in houses, in cars and on other related items are aimed to establish whether a contact with explosives has occurred. Such contact could indicate illegal possession or handling explosives or a possible involvement in shooting. Also, analysis of post explosion residues is one of the most technically difficult subjects in forensic science. The residual explosive has to be isolated from large amount of debris, often covering wide areas, and including large number of interfering items. In this work, in order to optimize a proper methodic way for post explosion analysis, artificial post explosion samples were created by mixing trace quantities of commonly used explosives with sand soil. Then sample extraction, filtration and concentration were carried out. Soil sample without any explosive traces was taken as a blank. The concentrated organic extract was then subjected to different available analytical chromatographic methods mainly HPLC, TLC, and spot test. Minimum detection limit of each technique was determined by gradually decreasing the amount of explosive residues in soil. Real explosions were carried out in the field, and post explosion samples were collected and investigated till complete identification of the exploded charge [1-5].
\end{abstract}

\section{KEY WORDS}

Analysis of explosives, Residual analysis, and Post explosion analysis

\section{NOMENCLATURE}

$\mathrm{R}_{\mathrm{f}}$

TLC

MDL

HPLC

$\mathrm{mAu}$

GC

ITMS

SPE

MDL
Rate of flow $=$ (distance traveled by the analyte) $/$ (distance traveled by the eluent)

Thin layer chromatography

Minimum detection limit

High performance liquid chromatography

Melli absorbane unit

Gas chromatography

Ion trap mobility spectrometry

Solid phase extraction technique.

Minimum detection limit

\footnotetext{
* Egyptian armed forces.
} 


\section{INTRODUCTION}

General comprehensive laboratory scheme for analysis of explosive residues was implemented in our post explosion work. The scheme includes screening of the debris items, clean-up procedure, extraction, and analysis. The organic extract often obtained using acetone is analyzed for organic explosives and related compounds. Post explosion analysis is usually characterized by small amount of residues present in complex highly contaminated matrix. Suitable clean up procedure could either be general or it could be designed to precede a specific analytical method. After clean up the extract is concentrated and subjected to a variety of analytical methods. The main steps that should be considered during post explosion analysis are summarized into:

\section{Sample collection.}

The best analytical methods will be useless if wrong items are collected. The unexploded explosives if present are usually not visible to naked eye, so that the successful collection of explosive residues depends upon taking the debris, which are most likely to contain them.

\section{Microscopic examination of debris.}

Residue sample can sometimes be identified visually by their characteristic appearance. Color and shape can give a clue to the general type or even to the particular explosive.

\section{Extraction of debris.}

Extraction of the debris transfers the explosive residues into solutions. In solution the explosives are easily subjected for further treatments like concentration, separation, and identification. The ratio of solvent to sample is usually in the range of $2-5 \mathrm{ml} / \mathrm{g}$.

\section{Sample recovery and clean up.}

Efficient clean up procedure for post explosion or hand swabs samples is an important perquisite for a successful analysis. The direct application of chromatographic methods (TLC, HPLC and GC) to sample in which the explosives constitute only a small part of a complex highly contaminated matrix is often unsuccessful in identifying the explosive. Suitable recovery or clean up procedures should there fore precede the chromatographic analysis. Most clean up procedure are based on adsorption of the explosives from organic extracts of hand swaps or debris on to solid adsorbent, and subsequent elution of the explosives from the adsorbent.

\section{Miscellaneous analytical methods.}

The extracts of post explosion debris, hand swabs, clothing or other samples containing explosive residues have been analyzed by various methods, often after some clean up. According to the type of the analyte, analytical methods are classified into two groups organic and inorganic [6-12]. 


\section{EXPERIMENTAL WORK}

In order to optimize proper way of extraction, filtration, concentration, sample clean up, and employing proper analytical techniques, post explosion samples were simulated by mixing trace quantities of common explosives with soil. Real explosions were carried out in the field, and real post explosion samples were investigated till complete identification of the exploded charge. The investigated explosives are: TNT, DNT, RDX, HMX, Tetryl, and PETN.

\section{1) Analysis of explosives residues in soil.}

Exactly about $100 \mathrm{gm}$ of soil sample with trace amount of all investigated explosives were mixed. Then sample extraction, filtration, and concentration were carried out. Soil sample without any explosive traces was taken as a blank. The employed techniques were repeated with ultra trace quantities of explosives with soil. The organic extract was concentrated up to $3 \mathrm{ml}$.

\section{a)Sample extraction.}

Extraction process was carried out by boiling sample under reflux with vigorous stirring. The procedure of extraction is carried out as follow:

1) Acetone was found to be the best solvent for all explosives, with low boiling point and good solving ability.

2) The amount of acetone suggested is about $3 \mathrm{ml} / 1 \mathrm{~g}$ sample.

3) Heating was carried out in water bath for 6 hours, with suitable heating rate to prevent over heating, or sample decomposition.

\section{b) Organic extract filtration and concentration.}

Proper filtration of soil is difficult due to blocking of the filter paper, and hence requires a strong vacuum pump. Sample concentration up to $3 \mathrm{ml}$ was achieved by simple fractional distillation. The sample was sucked by an analytical syringe and filtered by $0.2 \mu \mathrm{m}$ pore size filter.

\section{c) Analytical methods.}

The concentrated organic extract was subjected to chromatographic analytical methods mainly TLC and HPLC.

\section{HPLC analysis:}

The concentrated organic extract was analyzed by using Agilent HPLC 1100 series instrument, with the following specifications:

Column: $150 \mathrm{~mm}$ * $10 \mathrm{~mm}$ ld reversed phase column Zorbax SB C-18.

Mobile Phase:

Injection volume:

Detector:

Post run:

Column temperature:

Mobile Phase gradient:
$\mathrm{ACN}$ - Water.

$3 \mu \mathrm{l}$.

UV Detector at $220 \mathrm{~nm}$.

30 minute.

$28^{\circ} \mathrm{C}$ 


\begin{tabular}{|c|ccc|c|}
\hline$\%$ ACN & \multicolumn{3}{|c|}{ Time } & Flow rate \\
\hline $20 \%$ & $0:$ & 8 & $\mathrm{~min}$. & $0.3 \mathrm{ml} / \mathrm{min}$. \\
\hline $29 \%$ & $8.1:$ & 35 & $\mathrm{~min}$. & $0.3 \mathrm{ml} / \mathrm{min}$. \\
\hline $100 \%$ & $35.1:$ & 45 & $\mathrm{~min}$. & $0.5 \mathrm{ml} / \mathrm{min}$. \\
\hline
\end{tabular}

\section{TLC analysis.}

The concentrated organic extract was also analyzed by TLC technique using.

Mobile phase: $\quad$ Toluene - Cyclohexane (7:3)

Stationary phase: $\quad$ Meric aluminum sheet coated with silica gel 60 .

Developing agent: $\mathrm{NaOH} / \mathrm{Griess}$ reagent

\section{2) Real post explosion analysis.}

To perform such experimental work, unconfined $2 \mathrm{Kg}, 4 \mathrm{Kg}$ TNT charges, and shrapnel mines were exploded in the field. The site of explosion was examined by the aid of magnifying lens to locate unexploded charge. Samples were collected from debris, which suspected to contain unexploded explosive traces. Post explosion samples were collected from the explosion center. The laboratory scheme for post explosion analysis was implemented through proper sample collection, extraction, filtration, and clean up.

\section{a) Sample collection and extraction.}

- Appropriate sample collection is very important.

- Extraction process was carried out by boiling sample under reflux in water bath for 6 hours.

- About $300 \mathrm{gm}$ of soil sample in $2000 \mathrm{ml}$ extracting conical flask contains $900 \mathrm{ml}$ acetone

- All extraction procedures were carried out for clean soil sample without any explosives residues (blank sample).

\section{b) Sample filtration.}

Soil sample filtration is a very important step, as it helps in removing most of the solid particles from organic extract. Normally this soil sample is very fine, as it was subjected to pressure in the order of $10^{5}$ atmosphere. Therefore, making proper sample filtration is a difficult process, due to the blocking of the filter paper. This process requires a powerful vacuum pump. Filtration sequence is described below.

1) Filtration was carried out by Buchner funnel with strong vacuum pump. The organic extract was filtered again on a normal filter paper without vacuum.

2) The organic extract was then filtered by $0.4 \mu \mathrm{m}$ pore size omega thick filter paper made of cellulose. The application of this type of filter paper is considered to be the key step of sample purification.

3) Then the organic extract was filtered through $0.2 \mu \mathrm{m}$ pore size filter by using vacuum pump. 
4) The organic extract was concentrated up to $3 \mathrm{~mm}$, taken by an analytical syringe, and filtered through disposable $0.2 \mu \mathrm{m}$ pore size filter cartridge before the application of the analytical techniques.

\section{c) Sample concentration.}

Concentration was carried out by heating the organic extract on a water bath. Acetone was recovered in a clean conical flask. Some post explosion samples were concentrated up to $3 \mathrm{ml}$ and were studied using available analytical methods directly. Other samples were concentrated up to $10 \mathrm{ml}$ and were subjected to sample clean up by solid phase extraction technique (SPE), before the application of analytical methods.

\section{d) Sample clean up by solid phase extraction (SPE).}

The unreacted explosive traces that remain are usually very limited in quantity and are highly contaminated with various types of many interfering substances, which would interfere the analytical methods. At first sample clean up by liquid-liquid partition was investigated, this was achieved by evaporating acetone and using 100 $\mathrm{ml}$ benzene or diethyl ether as they aren't miscible with water. Then sample clean up was carried as follow:

a) Partition with $100 \mathrm{ml}$ solution of $5 \% \mathrm{NaOH}$ to remove acidic fraction.

b) Partition with $100 \mathrm{ml}$ solution of $5 \% \mathrm{HCL}$ to remove alkaline fraction.

c) Partition with $100 \mathrm{ml}$ distilled water to remove normal fraction.

This technique was found to be inefficient for the following reasons:

a) Both benzene and ether aren't good solvent for explosives.

b) Partition with $5 \% \mathrm{NaOH}$ causes decomposition to poly nitro aromatics.

c) Partition with $5 \% \mathrm{HCL}$ causes decomposition to Nitramines.

So, sample clean up by SPE technique was employed by using porapack RDX seppack (SPE) column. Porapack RDX column is the most sensitive technology available today and allows analysis of sub part per billion (sub ppb) levels. Porapak RDX solid phase extraction technique is shown in Fig.1. Porapack RDX column was employed first for standard mixtures of all investigated explosives in the organic extract of blank sample. These samples were prepared, in order to study the behavior of common organic explosives on the cartridge, in order to optimize proper operating conditions (to know how and when explosives are eluted), then the cartridge was employed for real post explosion samples.

\section{e) Procedure of SPE technique}

The recommended scheme for using Porapack RDX solid phase extraction cartridge is presented here.

1) Sample preparation:

- Standard mixture of common explosives of concentration $0.5 \mathrm{mg} / \mathrm{ml}$ of each explosive was prepared. This mixture was prepared by weighing exactly about $100 \mathrm{mg}$ of each explosive. The total weighed mixture was dissolved in $200 \mathrm{ml}$ acetone. The investigated explosives are: TNT, DNT, RDX, HMX, Tetryl, and PETN. 
- In order to determine the proper operating conditions. Artificial post explosion sample was created by mixing $2 \mathrm{ml}$ of standard explosives mixture with $2 \mathrm{ml}$ of organic extract of blank soil sample. The organic sample was mixed with $60 \mathrm{ml} \mathrm{H}_{2} \mathrm{O}$.

- The organic extract of real post explosion samples was concentrated up to $10 \mathrm{ml}$, and then it was mixed with $150 \mathrm{ml} \mathrm{H}_{2} \mathrm{O}$.

- Generally post explosion organic extract mixed with water in the ratio 1/15 volume ratio.

2) Cartridge conditioning.

Sep pack cartridge was activated as follow:

- Flow $15 \mathrm{ml} \mathrm{ACN}$ through the cartridge at $10 \mathrm{ml} / \mathrm{min}$.

- Flow $30 \mathrm{ml} \mathrm{H}_{2} \mathrm{O}$ through the cartridge at $10 \mathrm{ml} / \mathrm{min}$.

3) Sample loading.

The sample was loaded onto the cartridge at $2: 10 \mathrm{ml} / \mathrm{min}$.

4) Interfering elution.

The wash step removes interferences while retaining the analyte. In order to optimize washing method many samples was investigated as follow:

a) Standard mixture of artificial post explosion sample was washed with $15 \mathrm{ml}$ deionized water.

b) Other artificial post explosion sample was washed with $15 \mathrm{ml}$ hexane, and then with $15 \mathrm{ml}$ deionized water. It was found to be the best washing method, and it was employed for real post explosion samples.

c) Real post explosion sample of shrapnel mines was washed with $15 \mathrm{ml}$ hexane, and then with $15 \mathrm{ml}$ deionized water.

5) Analyte elution.

Analyte was eluted by $20 \mathrm{ml}$ ACN. The organic solution was concentrated up to $3 \mathrm{ml}$.

6) Recovery of SPE technique.

The recovery of SPE technique was calculated after eluting artificial post explosion sample by $20 \mathrm{ml}$ ACN. The organic extract was concentrated up to 3 $\mathrm{ml}$. The concentration of the eluted explosives was calculated by employing external standard method (EST) method to standard mixture. Recovery was calculated for artificial post explosion sample washed with $15 \mathrm{ml}$ de ionized water, and for artificial post explosion sample washed with $15 \mathrm{ml}$ Hexane, then with 15 $\mathrm{ml}$ de ionized water.

\section{f) Study the post explosion sample by available analytical methods.}

The available chromatographic techniques were employed for the analysis of concentrated post explosion sample dissolved in acetone. The analysis was performed for the investigation of post explosion samples before and after carrying sample clean up by (SPE). 


\section{g) Application of HPLC in post explosion analysis:}

The concentrated organic extract was analyzed by the aid of HPLC technique. The operating conditions are the same as for analysis of explosive residues with soil, except that the flow rate was $0.3 \mathrm{ml} / \mathrm{min}$. without change all over the experiment.

\section{II) Application of TLC in post explosion analysis (without SPE).}

The concentrated organic extract was analyzed by TLC technique, with the same operating conditions for explosive traces with soil.

\section{III) Application of available spot test for post explosion samples}

The extracted sample dissolved in acetone was subjected to TNT reagent which is $30 \% \mathrm{NaOH}$ solution.

\section{RESULTS AND DISCUSSIONS}

Many solvents were investigated for explosive debris analysis. Finally acetone was found to be the best recommended one, because it is a good solvent for all explosives, and it has low boiling point. The concentrated organic extract was then subjected to all available analytical methods, mainly HPLC and TLC to verify their sensitivity to post explosion sample, and to determine their minimum detection limit (MDL) expressed in ppm.

\section{1) Analysis of explosive residues with soil}

A concentrated organic extract of blank sample and a set of concentrated organic extracts of gradually decreased trace quantities of explosives with soil expressed in ppm and sub ppm were injected in sequence under the same operating conditions to determine their minimum detection limit.

\section{Application of HPLC for analysis of explosive residues in soil.}

HPLC technique was employed for investigation of explosive residues with soil.

From the blank chromatogram it was clear that many interfering substances, were extracted by acetone. For this reason sample clean up by solid phase extraction technique (SPE) in order to remove these interfering substances (mainly aliphatic peak at 7.721), would be an important step especially in heavily contaminated matrix. The obtained HPLC chromatogram of explosive residues with soil is shown in Fig.2. The minimum detection limit of explosives in soil expressed in ppm is shown in Table1.

\section{Application of TLC for explosive residues with soil.}

TLC chromatogram of all investigated explosives is shown in Fig.3. Rate of flow and minimum detection limit of all investigated explosives is given in Table 2. 


\section{2) Post explosion analysis.}

Field explosions were carried out for slightly buried $2 \mathrm{Kg}, 4 \mathrm{Kg}$ TNT charge. Also, demolition of buried Sharapnel mines was performed. Post explosion analysis scheme was implemented through proper sample collection, extraction, filtration, and concentration. The concentrated post explosion organic extracts were investigated by spot test and all available chromatographic analytical techniques including HPLC and TLC.

\section{Application of sample clean up by solid phase extraction (SPE).}

In order to study the main interfering peaks and to optimize sample clean up method, Artificial post explosion sample was prepared by mixing $2 \mathrm{ml}$ of the prepared standard mixture of common explosives with $2 \mathrm{ml}$ of the organic extract of blank sample. This mixture was investigated directly by HPLC technique. The main abundant interfering peaks are aliphatic fraction compounds at 7.663 and acetone peak at 1.72. The acetone peak was removed and the aliphatic peak was decreased by carrying sample washing with $15 \mathrm{ml}$ deionized water, as shown in Fig. 4. Finally complete removal of the aliphatic peak was achieved by washing the sample with 15 $\mathrm{ml}$ Hexane, and then with $15 \mathrm{ml}$ de ionized water as shown in Fig. 5.

\section{Recovery of SPE technique:}

Thus the best method for sample clean up was achieved by using $15 \mathrm{ml}$ Hexane in order to remove aliphatic fraction, then with $15 \mathrm{ml}$ de ionized water in order to remove polar fraction and all water soluble interfering substances. The analyte was then eluted with $20 \mathrm{ml} \mathrm{ACN}$. The organic extract was then concentrated up to about 3 $\mathrm{ml}$ before carrying HPLC investigation. For artificial post explosion samples after indicating best clean up method it is necessary to calculate recovery of this technique. This was achieved by HPLC technique, by employing HPLC external standard method (EST) to determine each eluted explosive concentration in $(\mathrm{ng} / \mu \mathrm{l})$. Recovery was calculated for sample washed with $15 \mathrm{ml}$ de ionized water, and for sample washed with $15 \mathrm{ml}$ Hexane, then with $15 \mathrm{ml}$ de ionized water. The obtained recovery result is given in Table 3 . From the previous results it is clear that complete sample clean up was achieved on the account of recovery. The obtained HPLC chromatogram of real explosion of Shrapnel mines after carrying complete sample clean up by elution with $15 \mathrm{ml}$ Hexane, then with $15 \mathrm{ml} \mathrm{H}_{2} \mathrm{O}$ is shown in Fig. 6. The chromatogram shows more stable base line, more obvious TNT peak and the obtained compound name and its concentration in $\mathrm{ng} / \mu \mathrm{l}$ by employing qualitative and quantitative identification by external standard technique (EST).

\section{Application of TLC in post explosion sample analysis.}

TLC technique was employed for the analysis of post explosion samples that were taken from the center of real explosion of shrapnel mines. About $20 \mu \mathrm{L}$ of these samples with standard TNT sample were investigated by TLC technique. The obtained TLC chromatogram is shown in Fig.7. 


\section{Application of spot methods for analysis of post explosion sample.}

Spot tests are the main chemical characteristics, which help in detection and identification of explosives. The identification by color reaction is based on the fact that the color produced is characteristic of a certain compound or a defined group of compounds. The sensitivity of most color reactions used in the analysis of explosives is in the range of $10^{-5}: 10^{-7} \mathrm{~g}$. Post explosion samples investigated by HPLC and TLC were also investigated by spot test employed for TNT detection. The same behavior of post explosion samples and standard TNT solution was given with $30 \% \mathrm{NaOH}$ solution by the formation of unstable Meizenheimer type complex, which give blueviolet color that, turns dark with time. This method provided more conformation about the exploded charge.

\section{CONCLUSION AND FUTURE WORK}

The presented work has revealed some important recommendations that should be considered during post explosion analysis.

- Examination of the site of explosion gives primary inspection of the type of exploded charge, as TNT explosion is characterized by the presence of gray color all over the site of explosion. This is could be attributed to its strong negative $\mathrm{O}_{b}$.

* Proper sample collection from the site of explosion is the most important step. This is because the best analytical methods become meaningless if wrong items are collected.

* Efficient sample filtration sequence is essential to protect the applied chromatographic HPLC automatic syringe and column from blocking.

* In post explosion analysis, extraction by Pressurized solvent extraction would be an efficient and rapid extraction technique.

* The application of ion mobility spectrometry (IMS) for its role in explosive detection is recommended. The explosives residues are collected on a membrane by filter by a special attachment on a house hold vacuum. Subsequent thermal desorption and analysis requires 5 seconds. Experimental results have determined the limits of detection for most common explosives to be approximately $200 \mathrm{pg}$ [13-15].

* The combination between HPLC-MS and HPLC-IMS would be powerful identification tool especially in post explosion analysis.

* GC method that over comes the disadvantages of HPLC methods, including high solvent usage, co elution, long time run, sample dilution, and low sensitivity, would be an important request, especially when employing the most powerful identification tool GC-MS with identification mass spectral library, in parallel with the most sensitive detector to explosives thermal energy analyzer (GC-TEA) [16]. 


\section{FIGURES AND TABLES}

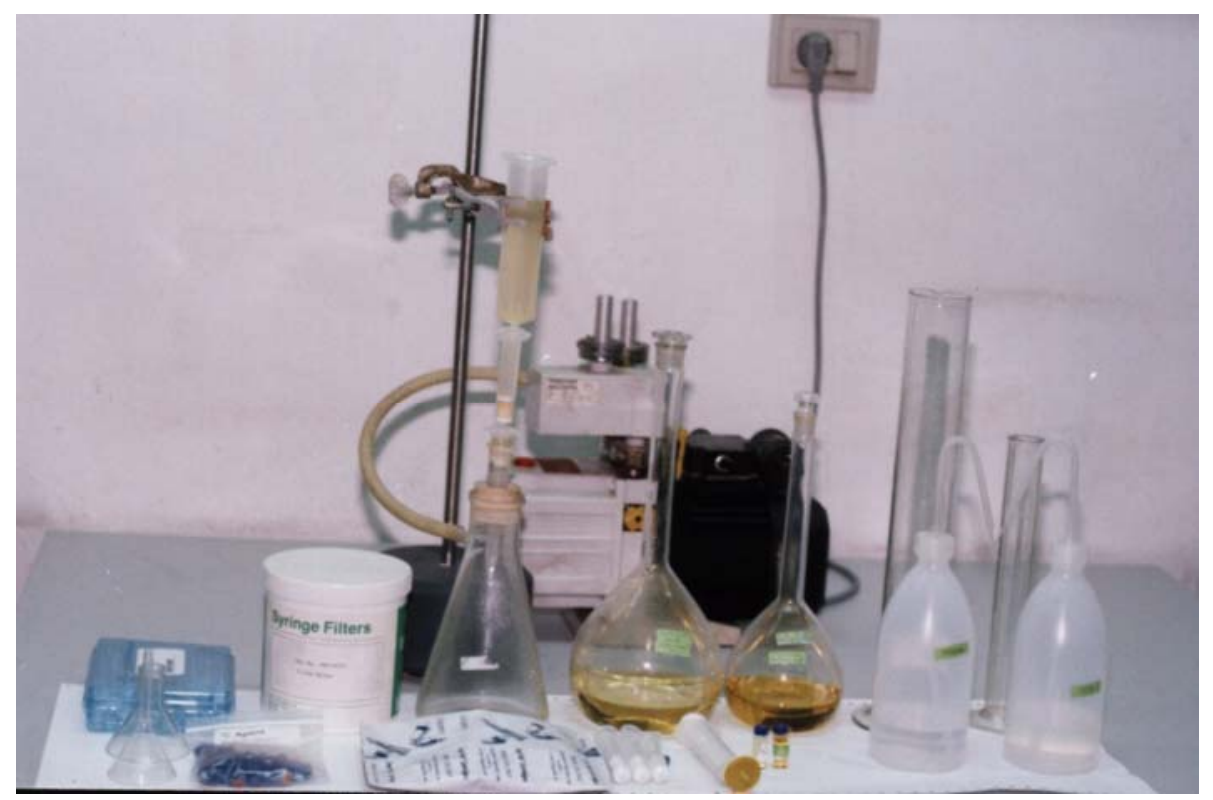

Fig.1 Porapak RDX solid phase extraction cartridge setup.

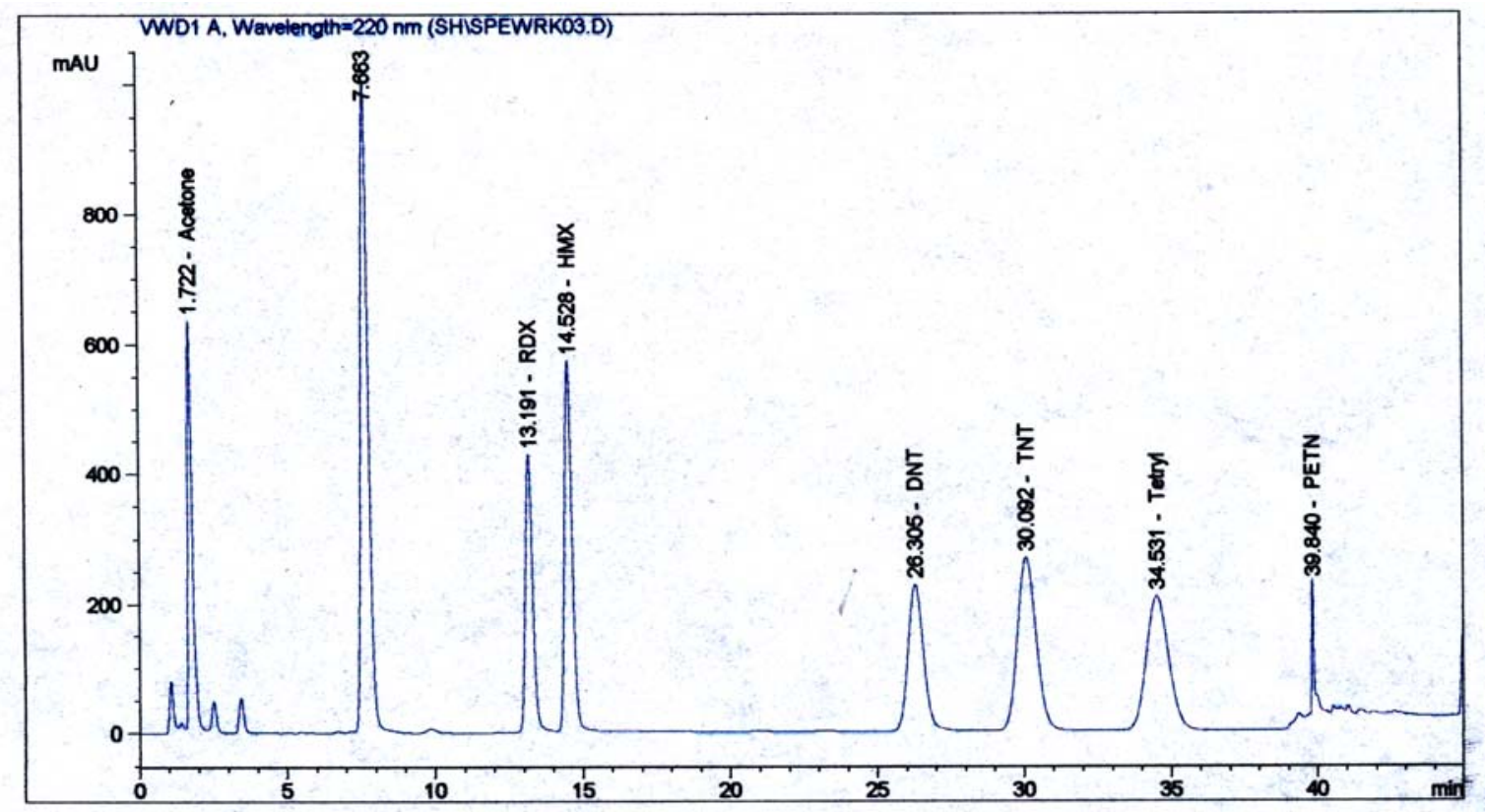

Fig.2 The output HPLC chromatogram of explosive trace residues in soil.

$\begin{array}{lllllll}\text { MIX. TNT PETN NG Tetryl RDX HMX NC } & \text { DNT }\end{array}$ 


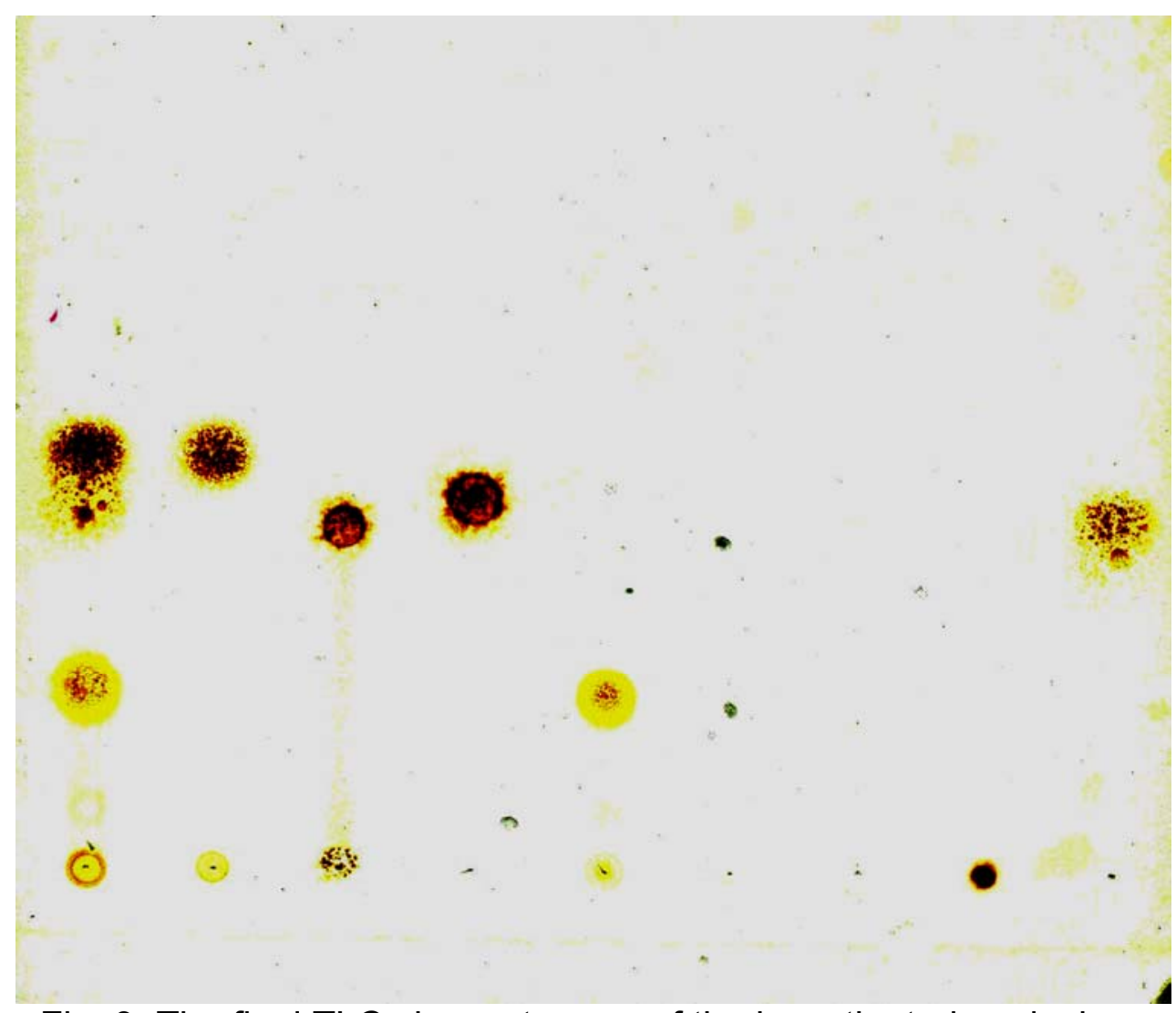

Fig. 3 The final TLC chromatogram of the investigated explosives

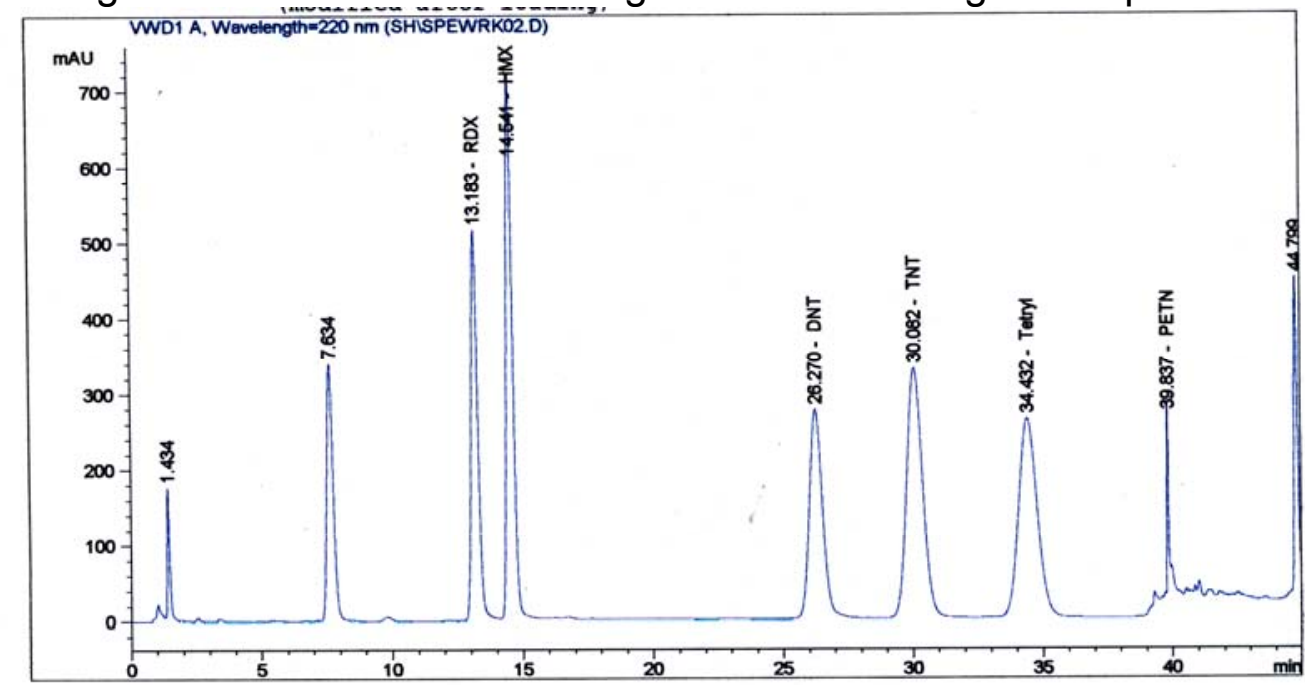

Fig. 4 HPLC chromatogram of simulated post explosion sample (after carrying sample washing with deionized water). 


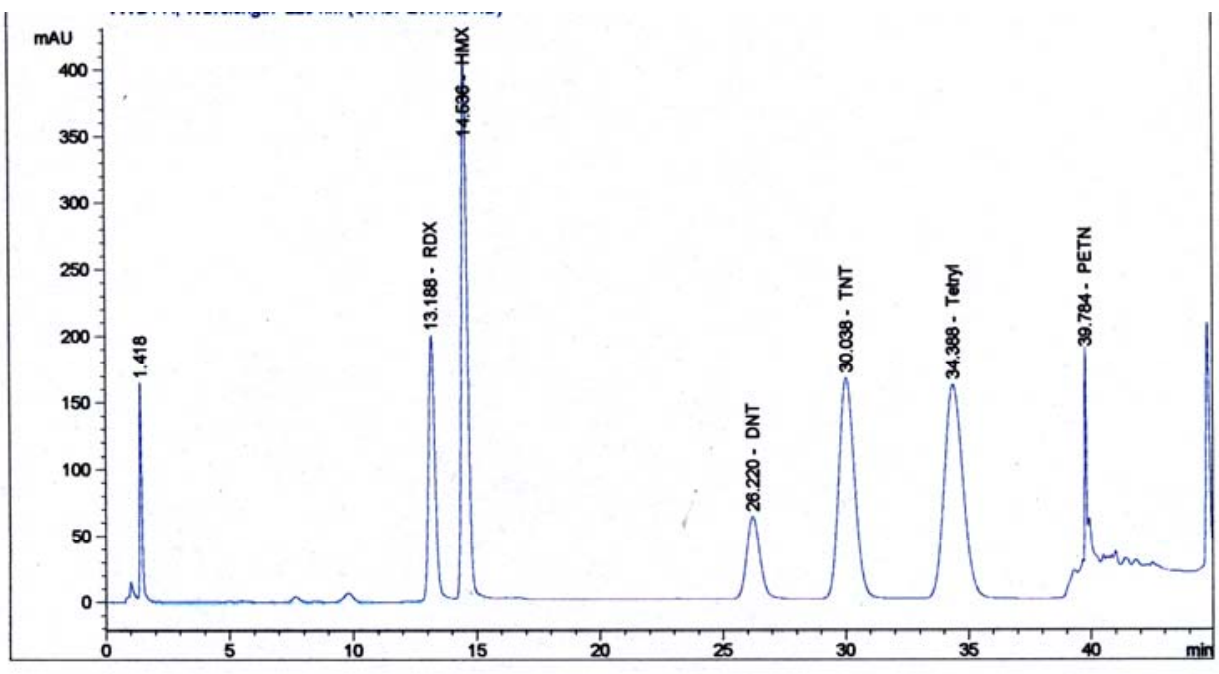

Fig. 5 HPLC chromatogram of simulated post explosion sample (after carrying sample washing with Hexane and deionized water).

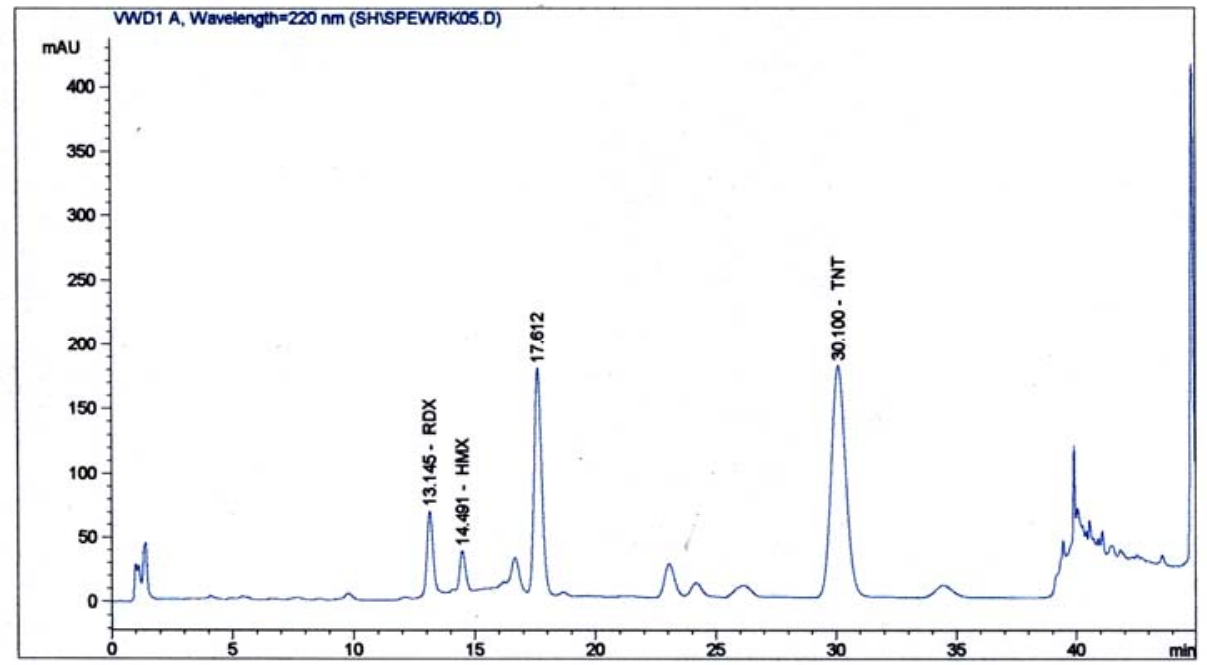

Fig. 6 Output HPLC chromatogram of post explosion sample of shrapnel mines after carrying sample clean up by (SPE).

Standard TNT Shrapnel mines $2 \mathrm{Kg}$ TNT 


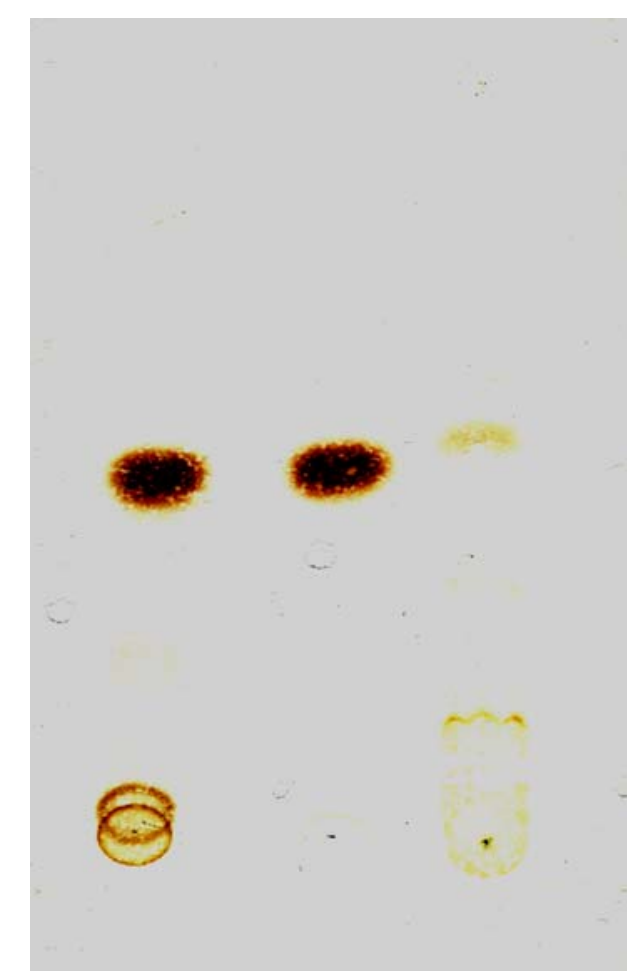

Fig.7 TLC Chromatogram for post explosion samples.

Table 1. HPLC MDL of explosive residues in soil.

\begin{tabular}{|c|c|c|}
\hline No. & Explosive & MDL ( PPm) \\
\hline 1 & TNT & 1 \\
2 & DNT & 1.3 \\
3 & RDX & 1.3 \\
4 & HMX & 1.3 \\
5 & Tetryl & 1 \\
6 & PETN & 2.5 \\
\hline
\end{tabular}

Table 2 TLC MDL of explosive residues in soil.

\begin{tabular}{|c|c|c|c|}
\hline No. & Explosive & Rate of flow $\left(\mathrm{R}_{\mathrm{f}}\right)$ & MDL ( PPm) \\
\hline 1 & TNT & 0.45 & 2 \\
2 & DNT & 0.38 & 5 \\
3 & RDX & 0 & 10 \\
4 & HMX & 0 & 15 \\
6 & Tetryl & 0.21 & 2.5 \\
\hline
\end{tabular}


Table 3 Recovery of SPE technique.

\begin{tabular}{|c|c|c|c|}
\hline No. & Explosive & $\begin{array}{c}\text { Recovery for clean up by } \\
(15 \mathrm{ml} \text { de ionized water })\end{array}$ & $\begin{array}{c}\text { Recovery for clean up by } \\
\left(15 \mathrm{ml} \text { Hexane, then } 15 \mathrm{H}_{2} \mathrm{O}\right)\end{array}$ \\
\hline 1 & RDX & $78 \%$ & $69 \%$ \\
2 & HMX & $76 \%$ & $73 \%$ \\
3 & DNT & $69 \%$ & $34 \%$ \\
4 & TNT & $79 \%$ & $52 \%$ \\
5 & Tetryl & $79 \%$ & $69 \%$ \\
6 & PETN & $100 \%$ & $99 \%$ \\
\hline
\end{tabular}

\section{REFRENCES}

[1] J.Yinon and S. Zitrin; "The analysis of explosives", 1 st edition, Pergamon press, Oxford, 1981.

[2] J.yinon and S. Zitrin ; "Modern methods and applications in analysis of explosives", Wiley, Chichester, 1993.

[3] R. Anderson; "Sample pre-treatment and separation", John Wiley \& Sons, London, 1986.

[4] T. Kishi and J. Nakamura; "A scheme for the analysis of explosives and explosive residues in japan", Proceeding of the 4 th international symposium on analysis and detection of explosives, Kluwer academic publisher, Septemper 7101992.

[5] Waters Corporation; "Chromatography columns and supplies catalog", New York, 2001-2002.

[6] M. Kaiser; "HPLC optimization of the separation of explosives and propellant components with an octadecyl phase by computer simulation", Magazine: Propellant, Explosives and pyrotechnics, 22-321-325 (1997).

[7] US Environmental protection agency; "Test methods for evaluating solid waste, Physical/Chemical methods. sw-846 update III", Office of solid waste , Washington, DC, 1997.

[8] Agilent corporation; "Under standing Chemistation catalog", Hewlett Packard, New York, 2000.

[9] K. Rubinson and J. Rubinson; "Contemporary instrumental analysis", Printice Hall, New York, 2000.

[10] C. H. Daniel; "Quantitative chemical analysis ", 5 th edition, W.H. Freeman, New York, 1999.

[11] T. Shibamoto; "Chromatographic analysis of environmental and food toxicants", California, 1998.

[12] M. Kaiser; "Optimization of the flow rate in high pressure liquid chromatography of explosives", Magazine: Propellant, Explosives and pyrotechnics; 21-226-270, 1996. 
[13] D. Dean; "Detection of explosives evidence by ion mobility spectrometry ", Proceeding of the 4 th international symposium on analysis and detection of explosives; September 7-10, Kluwer academic puplishers, 1992.

[14] G E Ion Track Corporation; "Total ion trap mobility spectrometry technical paper", Washington DC, 2002.

[15] G E Ion Track Corporation; "Total ion trap mobility spectrometry Itemiser 3 data sheet ", Washington DC, 2002.

[16] F. Rouessac and A. Rouessac;" Chemical analysis modern instrumentation methods and techniques", John willey, New York, 2000. 